\title{
Some Rational Vehicle Motions
}

\author{
J.M. Selig
}

\begin{abstract}
It is observed that the kinematic equations of many vehicles take the same form. This form is that the body-fixed velocity twist of the vehicle lies in a fixed screw-system of a particular type. The Cayley map can be used to pull-back these equations to the Lie algebra of the group of rigid-body motions. Rational solutions to the equations can be found by the method of undetermined coefficients. Since the Cayley map is a rational map, mapping these rational solutions back to the group gives rational rigid-body motions. A 3-parameter family of rational Frenet-Serret motions is found in this way. Multiplying these motions by a rational roll-motion gives a 4-parameter family of aeroplane motions.
\end{abstract}

Key words: Rational rigid-body motions, Cayley map, cars, aeroplanes.

\section{Introduction}

Many vehicles are modelled as non-holonomic systems. Usually the kinematic equations for a vehicle's motion have the form

$$
G(t)^{-1} \frac{d G(t)}{d t}=S_{B}
$$

here $G(t)$ lies in the group of rigid-body motions and represents the motion of the vehicle. The body fixed velocity twist $S_{B}$ is constrained to lie in some fixed screw system. The ubiquity of this type of model can be explained by the fact that for most vehicles the actuators are fixed with respect to the body and provide forces or torques along screws fixed in the body. Many examples exist, a few are listed below.

Bicycles and cars. Perhaps the motivating example and the most intensely studied one. It is of course, a planar problem so the analysis can be done in the group

Faculty of Business, London South Bank University, SE1 0AA, U.K. e-mail: seligjm@1sbu.ac.uk 
of planar rigid displacements, $S E(2)$. The body-fixed velocity of such a vehicle is restricted to a forward velocity in only one direction and a rotational velocity about a line perpendicular to the plane.

Aeroplanes, roller-coasters, autonomous underwater vehicles. These vehicles can translate forward, in a fixed direction relative to the vehicle, but can also roll, pitch and yaw. Hence the body-fixed velocity twist $S_{B}$, of such a vehicle lies in a particular 4-system of screws.

Needle steering. The problem of steering a long flexible needle or cannula with a bevelled tip is now well known in robotics. The motion of the needle tip is a Frenet-Serret motion (see below) but based on a curve with constant curvature. This means that $S_{B}$ for this system lies in a 2-system of screws. The kinematic equations for this problem can be found in [5], see also [3].

Frenet-Serret motions. Not the motion of a vehicle but it does have that same kinematic equation as given in (1). Here the body-fixed velocity twist $S_{B}$, lies in a particular 3-system of screws. This example is often studied in computer aided design but these motions have also been proposed as suitable motions for the end-effector of a manipulator arm. See also Bishop motions [3].

Many workers have studied the path-planning problem for such vehicles. Here a very simple view is taken. The idea is to plan rigid-body motions using simple interpolation schemes. It is well known that polynomial interpolation on the group of rigid motions is far from straightforward due to the non-linear nature of the group. Moreover, another problem arises, not all motions in the group satisfy the kinematic equation (1), for the vehicle. This work addresses the problem of finding simple families of motions satisfying the vehicle's kinematic equations. Such families need to be large enough to be able to interpolate a wide range of possible motions yet simple enough to be easy to work with especially for computers. For these reasons rational motions are preferred.

The following strategy is used to find such families of motions. First the Cayley map is used to pull-back the kinematic equation to $\mathbb{R}^{6}$. Since the Cayley map is a rational map, rational curves in $\mathbb{R}^{6}$ will be mapped to rational curves in the group. Then a rational anzatz is substituted into the equation to turn the differential equation into algebraic equations. Solutions of the resulting algebraic equations will produce motions with the desired properties.

\section{The $4 \times 4$ Cayley Map}

In classical differential geometry a standard problem is to reconstruct a space-curve from its curvature and torsion functions. The differential equations to be solved for this problem have exactly the same for as given in (1) above. However, $G$ lies in $S O(3)$, the group of rotations about a point. The classical approach to the problem is to make a substitution which turns the equation into a Riccati equation, see [1] for example. 
This approach generalises to other matrix groups. The substitution required is the standard Cayley map. Suppose $G \in \mathscr{G}$, the matrix Lie group. Let $S$ be a square matrix representing an element of the Lie algebra to $\mathscr{G}$. The Cayley map is given by,

$$
G=(I+S)(I-S)^{-1},
$$

see [2] for more details. Substituting this into the kinematic equation (1) gives,

$$
\frac{d S}{d t}=\frac{1}{2}(I+S) S_{B}(I-S)=\frac{1}{2}\left(I+S S_{B}-S_{B} S-S S_{B} S\right) .
$$

This is an example of a matrix Riccati equation. If $S_{B}$ is a known function of time then this equation can be solved numerically to simulate the motion of the vehicle.

Here $G \in S E$ (3),the group of proper rigid-body displacements in 3-dimensions. It is assumed that all that is known is that $S_{B}$ lies in a known screw-system; a linear subspace of the Lie algebra to $S E(3)$. In the $4 \times 4$ representation of the Lie algebra to $S E(3)$ a general twist can be written as,

$$
S=\left(\begin{array}{cc}
\Omega & \mathbf{v} \\
0 & 0
\end{array}\right)
$$

where $\Omega$ is the anti-symmetric $3 \times 3$ matrix corresponding to the angular velocity vector $\omega$. That is, $\Omega \mathbf{p}=\omega \times \mathbf{p}$ for any vector $\mathbf{p}$. The vector $\mathbf{v}$ is the linear velocity of the origin.

The motion within this system is usually specified by a number of control functions related to the physical actuators. To project out these control functions equation (3) can be rearranged to give,

$$
2(I+S)^{-1} \frac{d S}{d t}(I-S)^{-1}=S_{B}
$$

Next we write the equation using the adjoint representation of the group. In this representation twists are represented as 6-dimensional column vectors. These vector are usually written in a partitioned form with the first three components of $\mathbf{s}$ given by $\omega$ and the second three by $\mathbf{v}$. In this representation equation (5) becomes,

$$
F(\mathbf{s}) \frac{d \mathbf{s}}{d t}=\mathbf{s}_{B}
$$

where $F(\mathbf{s})$ is the matrix.

$$
F(\mathbf{s})=\frac{2}{1+|\omega|^{2}}\left(\begin{array}{cc}
I-\Omega & 0 \\
V \Omega-V & \left(1+|\omega|^{2}\right) I-\Omega+\Omega^{2}
\end{array}\right) .
$$

Here $V$ is the $3 \times 3$ anti-symmetric matrix corresponding to $\mathbf{v}$. This result can be found using the methods presented in [2].

Suppose $\mathscr{W}_{1}, \ldots \mathscr{W}_{n}$ are a set of linearly independent wrenches forming a basis of the dual space to screw-system that $\mathbf{s}_{B}$ lies in. That is $\mathscr{W}_{i}^{T} \mathbf{s}_{B}=0, i=1, \ldots, n$. 

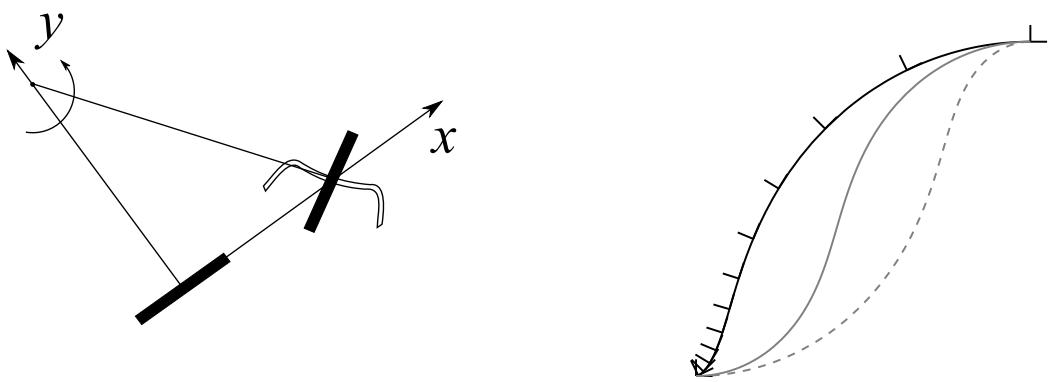

Fig. 1 Bicycle paths. The left-hand figure shows the body-fixed frame in the bike. On the right three different rational motions between the same positions are illustrated. The motion of the bodyfixed frame is shown for the top path.

Finally the kinematic equations can be written as,

$$
\mathscr{W}_{i}^{T} F(\mathbf{s}) \frac{d \mathbf{s}}{d t}=0, \quad i=i, \ldots, n .
$$

To make this clearer a simple example is presented in the next section.

\section{Rational Bicycle Motions}

This is a planar problem so let,

$$
S=\left(\begin{array}{ccc}
0 & -\omega & v_{x} \\
\omega & 0 & v_{y} \\
0 & 0 & 0
\end{array}\right) \quad \text { or as a column vector } \quad \mathbf{s}=\left(\begin{array}{c}
\omega \\
v_{x} \\
v_{y}
\end{array}\right) .
$$

With the coordinates given in fig. 1 the bicycle can translate in the $x$-direction or rotate about a vertical line located on the $y$-axis. In general any combination of these two infinitesimal motion is possible so that,

$$
\mathbf{s}_{B}=v\left(\begin{array}{l}
0 \\
1 \\
0
\end{array}\right)+\mu\left(\begin{array}{l}
1 \\
0 \\
0
\end{array}\right),
$$

where the arbitrary coefficients $v$ and $\mu$ are related to the forward velocity and turning velocity. The only wrench dual to every possible $\mathbf{s}_{B}$ is given by $\mathscr{W}^{T}=(0,0,1)$. This expresses the fact that the bicycle cannot instantaneously translate in the $y$ direction. In this planar problem the matrix $F(\mathbf{s})$ reduces to,

$$
F(\mathbf{s})=\frac{2}{1+\omega^{2}}\left(\begin{array}{ccc}
1 & 0 & 0 \\
-v_{y} & 1 & \omega \\
v_{x} & -\omega & 1
\end{array}\right)
$$


Hence the kinematic equation for the motion reduces to,

$$
v_{x} \dot{\omega}-\omega \dot{v}_{x}+\dot{v}_{y}=0 .
$$

We can produce polynomial solutions to this equation by choosing arbitrary polynomials for $\omega$ and $v_{x}$, substituting these into equation (11) and solving for $v_{y}$ by integration. For example, choosing $\omega=a_{1} t+a_{2} t^{2}$ and $v_{x}=b_{1} t+b_{2} t^{2}$ gives $v_{y}=(1 / 3)\left(a_{1} b_{2}-a_{2} b_{1}\right) t^{3}$. The constant terms, including the integration constant, have been chosen as zero, so that the motion begins at the identity when $t=0$. This gives a 4-parameter family of motions, with $a_{1}, a_{2}, b_{1}$ and $b_{2}$ as the 4-parameters.

The Cayley map can now be used to map this result to the $3 \times 3$ representation of the group,

$$
G_{P}(t)=\left(\begin{array}{ccc}
\frac{\left(1+a_{1} t+a_{2} t^{2}\right)\left(1-a_{1} t-a_{2} t^{2}\right)}{1+\left(a_{1}+a_{2} t^{2} t^{2}\right.} & \frac{-2\left(a_{1} t+a_{2} t^{2}\right)}{1+\left(a_{1}+a_{2} t\right)^{2} t^{2}} & \delta_{x} \\
\frac{2\left(a_{1} t+a_{2} t^{2}\right)}{1+\left(a_{1}+a_{2} t\right)^{2} t^{2}} & \frac{\left(1+a_{1} t+a_{2} t^{2}\right)\left(1-a_{1} t-a_{2} t^{2}\right)}{1+\left(a_{1}+a_{2} t\right)^{2} t^{2}} & \delta_{y} \\
0 & 0 & 1
\end{array}\right),
$$

where

$$
\begin{aligned}
& \delta_{x}=\frac{2\left(3 b_{1} t+3 b_{2} t^{2}-a_{1}\left(a_{1} b_{2}-a_{2} b_{1}\right) t^{4}-a_{2}\left(a_{1} b_{2}-a_{2} b_{1}\right) t^{5}\right)}{3\left(1+\left(a_{1}+a_{2} t\right)^{2} t^{2}\right)} \\
& \delta_{y}=\frac{2\left(3 a_{1} b_{1} t^{2}+\left(2 a_{1} b_{2}+4 a_{2} b_{1}\right) t^{3}+3 a_{2} b_{2} t^{4}\right)}{3\left(1+\left(a_{1}+a_{2} t\right)^{2} t^{2}\right)} .
\end{aligned}
$$

Examples of this motion are shown in Fig. 1.

\section{Rational Frenet-Serret Motions}

Frenet-Serret motions are usually defined with respect to a space-curve. Given a regular curve in space its Frenet-Serret motion is the motion of a body rigidly attached to the Frenet frame of the curve. This frame is sometimes known as the normaltangent-binormal coordinate system. The origin of the coordinate frame fixed in the body to be the point on the curve, the $x$-axis of the body-fixed frame will be aligned with the tangent to the curve and $y$-axis will be taken to lie along the curve's normal vector.

The motion of this frame can be written as a curve of group elements $G(t)$ satisfying the famous Frenet-Serret equations, which coincide with equation (1) above. In this case $S_{B}$ has the form,

$$
S_{B}=\left(\begin{array}{cccc}
0 & -v \kappa & 0 & 1 \\
v \kappa & 0 & -\nu \tau & 0 \\
0 & \nu \tau & 0 & 0 \\
0 & 0 & 0 & 0
\end{array}\right)
$$


where $v, \kappa$ and $\tau$ are respectively the speed, curvature and torsion of the curve generating the motion. The adjoint representation of this twist is,

$$
\mathbf{s}_{B}=\alpha\left(\begin{array}{l}
\mathbf{k} \\
\mathbf{0}
\end{array}\right)+\beta\left(\begin{array}{l}
\mathbf{i} \\
\mathbf{0}
\end{array}\right)+\gamma\left(\begin{array}{l}
\mathbf{0} \\
\mathbf{i}
\end{array}\right)
$$

where $\mathbf{i}, \mathbf{j}$ and $\mathbf{k}$ are the unit vectors in the $x, y$ and $z$-directions respectively and the coefficients $\alpha=v \kappa, \beta=\nu \tau$ and $\gamma=v$.

The three wrenches dual to any $\mathbf{s}_{B}$ are clearly given by,

$$
\mathscr{W}_{1}=\left(\begin{array}{l}
\mathbf{j} \\
\mathbf{0}
\end{array}\right), \quad \mathscr{W}_{2}=\left(\begin{array}{l}
\mathbf{0} \\
\mathbf{j}
\end{array}\right) \quad \text { and } \quad \mathscr{W}_{3}=\left(\begin{array}{l}
\mathbf{0} \\
\mathbf{k}
\end{array}\right) .
$$

Using the result for $F(\mathbf{s})$ from equation (7) above the three equations defining the motion can be given as,

$$
\begin{aligned}
0= & \dot{\omega}_{y}+\omega_{x} \dot{\omega}_{z}-\dot{\omega}_{x} \omega_{z} \\
0= & \left(\omega_{x} \omega_{y}-\omega_{z}\right) \dot{v}_{x}+\left(1+\omega_{y}^{2}\right) \dot{v}_{y}+\left(\omega_{x}+\omega_{y} \omega_{z}\right) \dot{v}_{z}+ \\
& \left(\dot{\omega}_{z}+\dot{\omega}_{x} \omega_{y}-\omega_{x} \dot{\omega}_{y}\right) v_{x}-\left(\dot{\omega}_{x}+\dot{\omega}_{y} \omega_{z}-\omega_{y} \dot{\omega}_{z}\right) v_{z} \\
0= & \left(\omega_{x} \omega_{z}+\omega_{y}\right) \dot{v}_{x}+\left(\omega_{y} \omega_{z}-\omega_{x}\right) \dot{v}_{y}+\left(1+\omega_{z}^{2}\right) \dot{v}_{z}- \\
& \quad\left(\dot{\omega}_{y}+\omega_{x} \dot{\omega}_{z}-\dot{\omega}_{x} \omega_{z}\right) v_{x}+\left(\dot{\omega}_{x}+\dot{\omega}_{y} \omega_{z}-\omega_{y} \dot{\omega}_{z}\right) v_{y} .
\end{aligned}
$$

Notice that the common denominators in these relations have been cancelled.

Now consider equation (17), this has exactly the same form as equation (11) above. Expressions for $\omega_{x}$ and $\omega_{z}$ can be chosen and then the solution for $\omega_{y}$ can be found by integration. Let us set $\omega_{x}=a t$ and $\omega_{z}=b t$ this gives $\omega_{y}=0$. To find possible solutions for $v_{x}, v_{y}$ and $v_{y}$ consider the anzatz,

$v_{x}=\alpha_{1} t+\alpha_{2} t^{2}+\alpha_{3} t^{3}+\alpha_{4} t^{4}, v_{y}=\beta_{1} t+\beta_{2} t^{2}+\beta_{3} t^{3}+\beta_{4} t^{4}, v_{z}=\gamma_{1} t+\gamma_{2} t^{2}+\gamma_{3} t^{3}+\gamma_{4} t^{4}$.

Substituting the chosen solution for $\omega_{i}$ and the anzatz for the $v_{j}$ into equations (18) and (19) gives a pair of polynomial equations in $t$. These equations have degree 4 and 5 in $t$ and the coefficients are linear in the undetermined coefficients $\alpha_{i}, \beta_{j}$ and $\gamma_{k}$. These polynomials must vanish for all values of $t$ hence the coefficients can be set equal to zero. This produces a set of 11 linear equations in the 12 unknowns. The system can be solved easily to give,

$$
v_{x}=\alpha_{1} t+\frac{1}{3} \alpha_{1}\left(a^{2}+2 b^{2}\right) t^{3}, \quad v_{y}=\frac{1}{6} \alpha_{1}\left(a^{2}+b^{2}\right) b t^{4}, \quad v_{z}=\frac{1}{3} \alpha_{1} a b t^{3} .
$$

These results give the entries of $S$ and hence, using the Cayley map, the corresponding $4 \times 4$ matrix function of $t$ can be calculated, 


$$
G_{F S}(t)=\left(\begin{array}{cccc}
\frac{1+\left(a^{2}-b^{2}\right) t^{2}}{1+\left(a^{2}+b^{2}\right) t^{2}} & \frac{-2 b t}{1+\left(a^{2}+b^{2}\right) t^{2}} & \frac{2 a b t^{2}}{1+\left(a^{2}+b^{2}\right) t^{2}} & \frac{2 c}{3}\left(3 t+\left(a^{2}-b^{2}\right)\right) t^{3} \\
\frac{2 b t}{1+\left(a^{2}+b^{2}\right) t^{2}} & \frac{1-\left(a^{2}+b^{2}\right) t^{2}}{1+\left(a^{2}+b^{2}\right) t^{2}} & \frac{-2 a t}{1+\left(a^{2}+b^{2}\right) t^{2}} & 2 b c t^{2} \\
\frac{2 a b t^{2}}{1+\left(a^{2}+b^{2}\right) t^{2}} & \frac{2 a t}{1+\left(a^{2}+b^{2}\right) t^{2}} & \frac{1-\left(a^{2}-b^{2}\right) t^{2}}{1+\left(a^{2}+b^{2}\right) t^{2}} & \frac{4}{3} a b c t^{3} \\
0 & 0 & 0 & 1
\end{array}\right)
$$

note that $\alpha_{1}$ has been replaced by $c$ in the above for neatness. This is a 3-parameter family of rational Frenet-Serret motions. It includes the rational Frenet-Serret motion found by Wagner and Ravani [4], which is reproduced by setting $a=b=c=$ $1 / \sqrt{2}$ and a change of the coordinate frame.

\section{Rational Aeroplane Motions}

In [3] it is proved that these general moving frame motions can be factored into the product of a Frenet-Serret motion with a pure rotation about the heading direction, the tangent to the curve. Hence all that is needed to produce a rational family of such motions is to compose a rational rotation with a family of rational Frenet-Serret motions, such as the family derived above.

The required pure rotation is about the $x$-axis in the body and can be written,

$$
G_{R}(t)=\left(\begin{array}{cccc}
1 & 0 & 0 & 0 \\
0 & \frac{1-\lambda^{2} t^{2}}{1+\lambda^{2} t^{2}} & \frac{-2 \lambda t}{1+\lambda^{2} t^{2}} & 0 \\
0 & \frac{2 \lambda t}{1+\lambda^{2} t^{2}} & \frac{1-\lambda^{2} t^{2}}{1+\lambda^{2} t^{2}} & 0 \\
0 & 0 & 0 & 1
\end{array}\right)
$$

the parameter $\lambda$ is essentially the rate of rotation. Combining this with the FrenetSerret motions given in (20) gives a 4-parameter family of rational Aeroplane motions,

$$
\begin{aligned}
& G_{A}(t)=G_{F S}(t) G_{R}(t)= \\
& \left(\begin{array}{cccc}
\frac{1+\left(a^{2}-b^{2}\right) t^{2}}{1+\left(a^{2}+b^{2}\right) t^{2}} & \frac{-2\left(b t-(2 a+\lambda) b \lambda t^{3}\right)}{\left(1+\left(a^{2}+b^{2}\right) t^{2}\right)\left(1+\lambda^{2} t^{2}\right)} & \frac{2(2+a) b t^{2}-a b \lambda^{2} t^{4}}{\left(1+\left(a^{2}+b^{2}\right) t^{2}\right)\left(1+\lambda^{2} t^{2}\right)} & a_{x} \\
\frac{2 b t}{1+\left(a^{2}+b^{2}\right) t^{2}} & \frac{1-\left(a^{2}+b^{2}+4 a \lambda+\lambda^{2}\right) t^{2}+\left(a^{2}+b^{2}\right) \lambda^{2} t^{4}}{\left(1+\left(a^{2}+b^{2}\right) t^{2}\right)\left(1+\lambda^{2} t^{2}\right)} & \frac{-2\left((a+\lambda) t-\left(a^{2}+b^{2}+a \lambda\right) \lambda t^{3}\right)}{1+\left(a^{2}+b^{2}\right) t^{2}} & a_{y} \\
\frac{2 a b t^{2}}{1+\left(a^{2}+b^{2}\right) t^{2}} & \frac{2\left((a+\lambda) t-\left(a^{2}-b^{2}+a \lambda\right) \lambda t^{3}\right)}{\left(1+\left(a^{2}+b^{2}\right) t^{2}\right)\left(1+\lambda^{2} t^{2}\right)} & \frac{1-\left(a^{2}-b^{2}+4 a \lambda+\lambda^{2}\right) t^{2}+\left(a^{2}-b^{2}\right) \lambda^{2} t^{4}}{\left(1+\left(a^{2}+b^{2}\right) t^{2}\right)\left(1+\lambda^{2} t^{2}\right)} & a_{z} \\
0 & 0 & 0 & 1
\end{array}\right),
\end{aligned}
$$

where,

$$
a_{x}=\frac{2 c}{3}\left(3 t+\left(a^{2}-b^{2}\right)\right) t^{3}, \quad a_{y}=2 b c t^{2}, \quad a_{z}=\frac{4}{3} a b c t^{3} .
$$




\section{Conclusion}

In this work a new class of rigid-body motion has been introduced. These vehicle motions include many well known types associated to various types of vehicle as well as some other motions of interest in Computer Aided Design. By unifying these different types of motion it is possible to find methods which can be applied to any of them. For example the Cayley substitution given in section 2, will transform the kinematic equations for any vehicle motion into a Riccarti equation. The rest of the paper gives another method that can be used for any of these vehicle motions. The problem addressed is to find rational motions satisfying the kinematic equations for the vehicle. In Robotics this has applications in path planning, in Computer Aided Design is has applications to motion interpolations for example.

The results given here are only intended to be indicative, the main purpose is to show that very similar methods can be used for rather different vehicles. However, it is not too difficult to see how the solutions presented could be extended to more practical techniques.

\section{Acknowledgments}

This work was has been much improved following the comment of the anonymous reviewers.

\section{References}

1. L.P. Eisenhart, 1909, A Treatise on the Differential Geometry of Curves and Surfaces, Ginn and Co. Boston.

2. J.M. Selig, 2007, "Cayley Maps for SE(3)", The International Federation of Theory of Machines and Mechanisms 12th World Congress, Besançon.

3. J.M. Selig, "Characterisation of Frenet-Serret and Bishop Motions with applications to Needle Steering", to appear Robotica.

4. M. Wagner and B. Ravani, 1997, "Curves with rational Frenet-Serret motion", Computer Aided Geometric Design, 15:79-101.

5. R. J. Webster III, J. S. Kim, N. J. Cowan, G. S. Chirikjian and A. M. Okamura, 2006, "Nonholonomic Modeling of Needle Steering", The International Journal of Robotics Research, 25(5-6):509-525. 